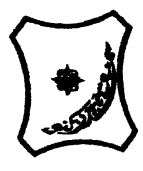

Bayero Journal of Pure and Applied Sciences, 10(1): 128 - 137

Received: November, 2016

Accepted: May, 2017

ISSN $2006-6996$

\title{
A MODIFIED RATIO-PRODUCT ESTIMATOR OF POPULATION MEAN USING SOME KNOWN PARAMETERS OF THE AUXILIARY VARIABLE
}

\author{
Umar Kabir Abdullahi and Abubakar Yahaya \\ Department of Statistics, Ahmadu Bello University, Zaria, Nigeria. \\ umarkabir9@gmail.com, +2348109554170. \\ abubakaryahaya@abu.edu.ng, +2348069334466.
}

\section{ABSTRACT}

The estimation of population mean is one of the challenging aspects in sampling theory and population study and much effort has been vigorously employed to improve the precision of estimates. In this research work, a modified ratio-product estimator of population mean $\bar{Y}$ of the study variable $Y$ using median and coefficient of variation of the auxiliary variable $X$, in simple random sampling scheme is proposed. The expression of bias and MSE of the proposed estimator have been obtained under large sample approximation, asymptotically optimum estimator (AOE) is identified with its approximate MSE formula. Estimator based on "estimated optimum values" was also investigated. Theoretical and empirical comparison of proposed estimator with some other ratio and product estimators justified the performance of the proposed estimators. $A$ minimum of 20 percent reduction in the MSE were observed from each of the existing estimators considered. It is found that the proposed estimator were uniformly better than all other modified ratio and product estimators and thus most preferred over the existing estimators for the use in practical application.

Keywords: Finite population mean, bias, mean square error, auxiliary variable, optimum estimator, study variable.

\section{INTRODUCTION}

The use of auxiliary variable (supplementary variable) has been widely discussed in sampling theory. Auxiliary variables are in use in survey sampling to obtain improved sampling designs and to achieve more precision in the estimates of some population parameters such as the mean, or the variance of the variable under study. This information may be use at both the stages of designing a survey (design, execution and estimation stages). It is well established that ratio, linear regression, product are some good examples in this context.

The estimation of population parameters, particularly mean is one of the challenging aspects in sampling theory and much effort has been vigorously employed to improve the precision of estimates. In sample survey literature, great classes of techniques for using auxiliary information by means of ratio, regression and product methods have been discussed in the presence of single and/or multi-auxiliary variables. A wide variety of estimators were proposed following distinct notions; different possible linear combinations and mixing together ratio, product, and/or linear regression estimators, each one exploiting the variables one at a time. It is also established that, if the regression line of the variable under study and the auxiliary variable passes through the origin and are positively correlated the best estimator to be used is ratio estimator (Singh and Espejo, 2003). In the same vein, if the regression line of the variable under study and the auxiliary variable pass through the origin but are negatively correlated the best estimator to be used is product estimator. On the other hand when the regression line does not passes through the origin but makes an intercept along the $y$-axis and there is correlation either positive or negative between the auxiliary variable $(X)$ and the variable of interest $(Y)$ the best estimator to use is the linear regression estimator. It is observed though, that all these methods yield biased estimators and of course bias decreases with increase in sample size (Okafor, 2002).

\section{BACKGROUND OF THE STUDY}

Consider a finite population $P=\left(P_{1}, P_{2}, \ldots, P_{N}\right)$ of $N$ units, let a sample be drawn using simple random sampling without replacement (SRSWOR), let $y_{i}$ and $x_{i}$ represent the values of a response variable $y$ and auxiliary variable $x$ respectively. The units of the finite population are identifiable in the sense that they are uniquely labeled from 1 to $N$ and the label on each unit is known. Further, suppose in a sample survey problem, we are interested in estimating the population mean $\bar{Y}$ of $y$, when the population parameters of the auxiliary variable $X$ such as population mean $\bar{X}$, knowledge of coefficient variation $C_{x}$, the coefficient of skewness $\beta_{1}(x)$, coefficient of Kurtosis $\beta_{2}(x)$, median $M_{d}$ of auxiliary variable $x$ are known. Before discussing further about the modified ratio estimators and the proposed modified ratio estimator, the notation used in this research work are described below. 
- $\quad N_{-}$- population size

- $n$-sample size

- $f=n / N^{- \text {sampling fraction }}$

- $\quad Y$ - study variable

- $X$ - auxiliary variable

- $\bar{Y}, \bar{X}$-population means

- $\bar{y}, \bar{x}$-sample means

- $S_{y}, S_{x}$ - sample standard deviations

- $C_{y}, C_{x}$-co-efficient of variations

- $\rho$-co-efficient of correlation

- $\quad \beta_{1}=\frac{N \sum_{i=1}^{N}\left(x_{i}-\bar{X}\right)^{3}}{(N-1)(N-2) S^{3}}$, coefficient of skewness of the auxiliary variable

- $\beta_{2}=\frac{N(N+1) \sum_{i=1}^{N}\left(x_{i}-\bar{X}\right)^{4}}{(N-1)(N-2)(N-3) S^{4}}-\frac{3(N-1)^{2}}{(N-2)(N-3)}$, coefficient of Kurtosis of the auxiliary variable

- $\quad M_{d}$ - median of the auxiliary variable

- $B($.$) - bias of the estimator$

- $M S E($.$) - mean square error of the estimator$

The traditional ratio and product estimators for $\bar{Y}$ are respectively given as,

$$
\begin{aligned}
& T_{R 1}=\bar{y}\left(\frac{\bar{X}}{\bar{x}}\right) \\
& T_{P 1}=\bar{y}\left(\frac{\bar{x}}{\bar{X}}\right)
\end{aligned}
$$

To the first degree of approximation the bias and mean squared errors of $T_{R 1}, T_{P 1}$ are respectively as,

$$
\begin{aligned}
& \operatorname{bias}\left(T_{R 1}\right)=\frac{(1-f)}{n} \bar{Y}\left[C_{x}^{2}\{1-K\}\right] \\
& \operatorname{bias}\left(T_{P 1}\right)=\frac{(1-f)}{n} \bar{Y} K C_{x}^{2} \\
& \operatorname{MSE}\left(T_{R 1}\right)=\frac{(1-f)}{n} \bar{Y}^{2}\left[C_{y}^{2}+C_{x}^{2}\{1-2 K\}\right] \\
& \operatorname{MSE}\left(T_{P 1}\right)=\frac{(1-f)}{n} \bar{Y}^{2}\left[C_{y}^{2}+C_{x}^{2}\{1+2 K\}\right]
\end{aligned}
$$

The modified ratio and product estimator by Subramani and Kumarapandiyan (2012) of $\bar{Y}$ are respectively given as,

$$
\begin{aligned}
T_{R 2} & =\bar{y}\left(\frac{\bar{X} C_{x}+M_{d}}{\bar{x} C_{x}+M_{d}}\right) \\
T_{P 2} & =\bar{y}\left(\frac{\bar{x} C_{x}+M_{d}}{\bar{X} C_{x}+M_{d}}\right)
\end{aligned}
$$


To the first degree of approximation the bias and mean squared errors of $T_{R 2}, T_{P 2}$ are respectively as,

$$
\begin{aligned}
& \operatorname{bias}\left(T_{R 2}\right)=\frac{(1-f)}{n} \bar{Y}\left[\theta C_{x}^{2}\{\theta-K\}\right] \\
& \operatorname{bias}\left(T_{R 2}\right)=\frac{(1-f)}{n} \bar{Y}\left[\theta C_{x}^{2}\{\theta-K\}\right] \\
& \operatorname{MSE}\left(T_{R 2}\right)=\frac{(1-f)}{n} \bar{Y}^{2}\left[C_{y}^{2}+\theta C_{x}^{2}\{\theta-2 K\}\right] \\
& \operatorname{MSE}\left(T_{P 2}\right)=\frac{(1-f)}{n} \bar{Y}^{2}\left[C_{y}^{2}+\theta C_{x}^{2}\{\theta+2 K\}\right] \\
& \text { where } \theta=\frac{\bar{X} C_{x}}{\bar{X} C_{x}+M_{d}}
\end{aligned}
$$

\section{Proposed estimator}

Motivated by Housila and Neha-Agnihotri (2008) and Subramani and Kumarapandiyan (2012), we defined a ratioproduct estimator of population mean using coefficient of variation and median of the auxiliary variable in simple random sampling as

$$
T_{R P}=\left[\delta \bar{y}\left(\frac{\bar{X} C_{x}+M_{d}}{\bar{x} C_{x}+M_{d}}\right)+(1-\delta) \bar{y}\left(\frac{\bar{x} C_{x}+M_{d}}{\bar{X} C_{x}+M_{d}}\right)\right]
$$

Where $\bar{y}=\frac{1}{n} \sum_{i=1}^{n} y_{i}$ and $\bar{x}=\frac{1}{n} \sum_{i=1}^{n} x_{i}$ are unbiased estimators of the population means $(\bar{Y}, \bar{X})$ respectively, where ' $C_{x}$ ' coefficient of variation and ' $M_{d}$ ' median are the known characteristics positive scalars of the auxiliary variable $X$ respectively and $\delta$ is a real constant to be determined such that the mean square error of $T_{R P}$ is minimum. The family of estimators $T_{R P}$ reduces to the following set of known estimators,

(i) For $\left(C_{x}, M_{d}, \delta\right)=(0,1, \delta), T_{R P} \rightarrow \bar{y}$ (usual unbiased estimator)

(ii) For $\left(C_{x}, M_{d}, \delta\right)=(0,1, \delta), T_{R P} \rightarrow \mathrm{T}_{1}=\left[\delta \bar{y}\left(\frac{\bar{X}}{\bar{x}}\right)+(1-\delta) \bar{y}\left(\frac{\bar{x}}{\bar{X}}\right)\right]$

which is due to Singh and Ruiz Espejo (2003)

(iii) For $\left(C_{x}, M_{d}, \delta\right)=\left(1, C_{x}, \delta\right), T_{R P} \rightarrow \mathrm{T}_{2}=\left[\delta \bar{y}\left(\frac{\bar{X}+C_{x}}{\bar{x}+C_{x}}\right)+(1-\delta) \bar{y}\left(\frac{\bar{x}+C_{x}}{\bar{X}+C_{x}}\right)\right]$

envisaged by Singh and Tailor (2005), where $C_{x}$ is the known population coefficient of variation and $M_{d}$ is the median of the auxiliary $X$ respectively, many other ratio-product estimators can be generated from $T_{R \mathrm{P}}$ by putting any suitable parameters rather than values of $\left(C_{x}, M_{d}, \delta\right)$.

\section{bias and Mean Square Error}

To obtain the bias and mean square error (MSE) of the proposed estimator $T_{R P}$ in (3.1), we write

Let $e_{0}=\frac{(\bar{y}-\bar{Y})}{\bar{Y}}$ and $e_{1}=\frac{(\bar{x}-\bar{X})}{\bar{X}}$

Then $\bar{y}=\left(1+e_{0}\right)$ and $\bar{x}=\left(1+e_{1}\right)$

Such that $E\left(e_{0}\right)=E\left(e_{1}\right)=0$

$$
\begin{aligned}
& E\left(e_{0}^{2}\right)=\frac{1-f}{n} C_{y}^{2}, E\left(e_{1}^{2}\right)=\frac{1-f}{n} C_{x}^{2}, E\left(e_{0} e_{1}\right)=\frac{1-f}{n} K C_{x}^{2} \\
& \text { Where } f=\frac{n}{N}, K=\rho \frac{C_{y}}{C_{x}}, \rho=\frac{S_{x y}}{S_{x} S_{Y}}, C_{y}=\frac{S_{y}}{\bar{Y}}, C_{x}=\frac{S_{x}}{\bar{X}} .
\end{aligned}
$$


Bajopas Volume 10 Number 1 June, 2017

$S_{x y}=\frac{\sum_{i=1}^{N}\left(x_{i}-\bar{X}\right)\left(y_{i}-\bar{Y}\right)}{(N-1)}, S_{x}^{2}=\frac{\sum_{i=1}^{N}\left(x_{i}-\bar{X}\right)^{2}}{(N-1)}, S_{y}^{2}=\frac{\sum_{i=1}^{N}\left(y_{i}-\bar{Y}\right)^{2}}{(N-1)}$.

And expanding (3.1) in terms of e's, we have

$T_{R P}=\bar{Y}\left(1+e_{0}\right)\left[\delta\left(1+\theta e_{1}\right)^{-1}+(1-\delta)\left(1+\theta e_{1}\right)\right]$

We assume that $\left|\theta e_{1}\right|<1$, so that the expression $\left(1+\theta e_{1}\right)^{-1}$ can be expanded to a convergent infinite series using binomial theorem. Hence from (3.2) we have.

$$
\begin{aligned}
& T_{R P}=\bar{Y}\left(1+e_{0}\right)\left[\delta\left(1-\theta e_{1}+\theta^{2} e_{1}^{2}-\theta^{3} e_{1}^{3}+\theta^{4} e_{1}^{4} \ldots\right)+(1-\delta)\left(1+\theta e_{1}\right)\right] \\
& =\bar{Y}\left[\delta\left(1+e_{0}\right)\left(1-\theta e_{1}+\theta^{2} e_{1}^{2}-\theta^{3} e_{1}^{3}+\theta^{4} e_{1}^{4} \ldots\right)+(1-\delta)\left(1+e_{0}\right)\left(1+\theta e_{1}\right)\right] \\
& =\bar{Y}\left[\delta\left(1+e_{0}-\theta e_{1}+\theta^{2} e_{1}^{2}-\theta e_{1} e_{0}+\theta^{2} e_{1} e_{0}+\theta^{2} e_{1}^{2} e_{0}-\theta^{3} e_{1}^{3}+\theta^{4} e_{1}^{4}-\theta^{3} e_{1}^{3} e_{0}+\ldots\right)+(1-\delta)\left(1+e_{0}+\theta e_{1}+\theta e_{1} e_{0}\right)\right] \\
& =\bar{Y}\left[1+e_{0}+\theta e_{1}+\theta e_{1} e_{0}+\delta\left(1+e_{0}-\theta e_{1}-\theta e_{1} e_{0}+\theta^{2} e_{1}^{2}+\theta^{2} e_{1}^{2} e_{0}-\theta^{3} e_{1}^{3}+\theta^{4} e_{1}^{4}-\theta^{3} e_{1}^{3} e_{0}+\ldots-1-e_{0}-\theta e_{1}-\theta e_{0} e_{1}\right)\right] \\
& =\bar{Y}\left[1+e_{0}+\theta e_{1}+\theta e_{1} e_{0}+\delta\left(-2 \theta e_{1} e_{0}-2 \theta e_{0} e_{1}+\theta^{2} e_{1}^{2}+\theta^{2} e_{1}^{2} e_{0}-\theta^{3} e_{1}^{3}+\theta^{4} e_{1}^{4}-\theta^{3} e_{1}^{3} e_{0}+\ldots\right)\right] \\
& =\bar{Y}\left[1+e_{0}-(1-2 \delta) \theta e_{1}+(1-2 \delta) \theta e_{1} e_{0}+\delta \theta^{2} e_{1}^{2}+\delta\left(\theta^{2} e_{1}^{2} e_{0}-\theta^{3} e_{1}^{3}+\theta^{4} e_{1}^{4}-\theta^{3} e_{1}^{3} e_{0}+\ldots\right)\right],
\end{aligned}
$$

We assume that the contribution of terms involving powers in $e_{0}$ and $e_{1}$ higher than the second is negligible, being of order $1 / n^{v}$, where $v>1$. Thus, from the above expression we write to a first order of approximation,

$$
\begin{aligned}
& T_{R P} \cong \bar{Y}\left[1+e_{0}-(1-2 \delta) \theta e_{1}+(1-2 \delta) \theta e_{1} e_{0}+\delta \theta^{2} e_{1}^{2}+\delta \theta^{2} e_{1}^{2} e_{0}\right], \text { or } \\
& \left(T_{R P}-\bar{Y}\right)=\bar{Y}\left[e_{0}-(1-2 \delta) \theta e_{1}+(1-2 \delta) \theta e_{1} e_{0}+\delta \theta^{2} e_{1}^{2}+\delta \theta^{2} e_{1}^{2} e_{0}\right],
\end{aligned}
$$

Taking the expectation of both side of (3.3), we obtained the bias of $\left(T_{R P}\right)$ to the first degree of approximation as

$\operatorname{bias}\left(T_{R P}\right)=\frac{(1-f)}{n} \theta \bar{Y}[K+\delta(\theta-2 K)] C_{x}^{2}$

will vanishes if

$\delta=\frac{K}{(2 \mathrm{~K}-\theta)}$

Thus for $\delta=\frac{K}{(2 \mathrm{~K}-\theta)}, T_{R P}$ is almost unbiased.

Squaring both side of the equation (3.3), and neglecting the terms of $e^{\prime} s$ having power greater than two we have

$$
\left(T_{R P}-\bar{Y}\right)^{2}=\bar{Y}^{2}\left[e_{0}^{2}+(1-2 \delta) \theta\left\{(1-2 \delta) \theta e_{1}^{2}+2 e_{1} e_{0}\right\}\right]
$$

Taking the expectation of both sides of (3.4), we get the mean square error MSE of $T_{R \mathrm{P}}$ to the first order of approximation as

$$
\operatorname{MSE}\left(T_{R P}\right)=\frac{(1-f)}{n} \bar{Y}^{2}\left[C_{y}^{2}+\theta(1-2 \delta) C_{x}^{2}\{(1-2 \delta) \theta+2 K\}\right]
$$

To obtain the value of $\delta$ that minimizes the $M S E$ of $\left(T_{R P}\right)$, we take the partial derivative of the $M S E$ of $\left(T_{R P}\right)$ with respect to $\delta$ and equate it to zero.

$\delta=\frac{1}{2}\left(1+\frac{K}{\theta}\right)=\delta_{0}$ (Optimum Value)

Putting (3.8) in (3.1), we get the Asymptotically Optimum Estimator (AOE) as

$T_{R P O}=\frac{\bar{y}}{2}\left[\left(1+\frac{K}{\theta}\right)\left(\frac{\bar{X} C_{x}+M_{d}}{\bar{x} C_{x}+M_{d}}\right)+\left(1-\frac{K}{\theta}\right)\left(\frac{\bar{x} C_{x}+M_{d}}{\bar{X} C_{x}+M_{d}}\right)\right]$

Substitution of (3.8) in (3.7) yield the minimum $M S E$ of $\left(T_{R P}\right)$ or the $M S E$ of asymptotically optimum estimator $(A O E) T_{R P}$ as 
Bajopas Volume 10 Number 1 June, 2017

$M S E_{\text {min }}\left(T_{R P}\right)=\frac{(1-f)}{n} S_{y}^{2}\left(1-\rho^{2}\right)=\operatorname{MSE}\left(T_{R P O}\right)$

which is equal to the approximate $M S E$ of regression estimator

$\bar{y}_{l r}=\bar{y}+\hat{\beta}(\bar{X}-\bar{x})$

Where $\hat{\beta}=s_{x y} / s_{x}^{2}$, is the sample estimate of the population regression coefficient $\beta$ of $y$ on $x$.

$s_{x y}=\frac{\sum_{i=1}^{n}\left(x_{i}-\bar{x}\right)\left(y_{i}-\bar{y}\right)}{(n-1)}, s_{x}^{2}=\frac{\sum_{i=1}^{n}\left(x_{i}-\bar{x}\right)^{2}}{(n-1)}$.

It is to be noted the $A O E$ of $\left(T_{R P O}\right)$ in (3.9) depends on $K$ and $\theta$, so the $A O E$ of $\left(T_{R P O}\right)$ can be used in practice only when $K$ and $\theta$ are known. Here it should be mentioned that $\theta$ is a function of known quantities $\left(C_{x}, M_{d}, \delta\right)$. So only the value of $K$ should be known for making the use of $A O E$ of $\left(T_{R P O}\right)$ in practice. The value of $K$ can be made known quite accurately either from pilot study or past data or experience gathered in due course of time. This problem has been discussed among others by Murthy (1967), Reddy (1978), Srivankataramana and Tracy (1980). Thus, the value of $K$ can be guessed quite accurately and such an estimator can be used in practice.

\section{Allowable Departure}

Let $k_{0}$ be an estimate or guessed value of $K$ with

$k_{0}=K(1+\eta)$, then

$\delta=\frac{1}{2}\left(1+\frac{K}{\theta}\right)=\frac{1}{2}\left(1+\frac{K}{\theta}+\frac{1}{\theta}\left(k_{0}-K\right)\right)=\delta_{0}+\frac{\eta K}{2 \theta}$

Putting (4.1) in (3.7) we obtain the $M S E$ of $\left(T_{R P}\right)$ as

$$
\begin{aligned}
& \operatorname{MSE}\left(T_{R P}\right)=\left(T_{R P O}\right)+\left(\frac{1-f}{n}\right) \rho^{2} \eta^{2} S_{y}^{2} \\
& \Rightarrow \operatorname{MSE}\left(T_{R P}\right)-\operatorname{MSE}\left(T_{R P O}\right)=\left(\frac{1-f}{n}\right) \rho^{2} \eta^{2} S_{y}^{2} \\
& \Rightarrow \frac{\operatorname{MSE}\left(T_{R P}\right)-M S E\left(T_{R P O}\right)}{M S E\left(T_{R P O}\right)}=\left(\frac{\rho^{2} \eta^{2}}{\left(1-\rho^{2}\right)}\right)
\end{aligned}
$$

It follows from $(3.25)$ that the proportional increase in $M S E$ of $\left(T_{R P}\right)$ over that of $A O E$ of $\left(T_{R P}\right)$ is less than $\gamma$ if,

$$
\begin{gathered}
\frac{\rho^{2} \eta^{2}}{\left(1-\rho^{2}\right)}<\gamma \\
\text { i.e. }|\eta|<\sqrt{\frac{\left(1-\rho^{2}\right)}{\rho^{2}} \gamma^{\prime}}
\end{gathered}
$$

Which clearly shows that to ensure only a small relative increase in $\operatorname{MSE}$ of $\left(T_{R P}\right),|\eta|$ must be in the neighborhood of "zero" if $\rho$ is high but can depart substantially from "zero" if $\rho$ is moderate.

\section{Efficiency Comparisons}

In order to compare the efficiency of the proposed a new ratio-product estimator with the various existing estimators we require the expressions of the MSE of the estimators, up to the first order of approximation. It is well known that under SRSWOR that

$\operatorname{Var}(\bar{y})=\left(\frac{1-f}{n}\right) S_{y}^{2}$

From (3.7) and (5.1) we have

$\operatorname{Var}(\bar{y})-\operatorname{MSE}\left(T_{R P}\right)=\left(\frac{1-f}{n}\right) \theta \bar{Y}^{2} C_{x}^{2}(2 \delta-1)[(1-2 \delta) \theta+2 K]$

which is non- negative if

$\min \left\{\frac{1}{2}, \frac{1}{2}\left(1+\frac{2 K}{\theta}\right)\right\}<\delta<\max \left\{\frac{1}{2}, \frac{1}{2}\left(1+\frac{2 K}{\theta}\right)\right\}$ 
Bajopas Volume 10 Number 1 June, 2017

It is to be noted that for $\delta=1$, the estimator $T_{R P}$ reduces to the ratio-type estimator

$T_{R 2}=\bar{y}\left(\frac{\bar{X} C_{x}+M_{d}}{\bar{x} C_{x}+M_{d}}\right)$

where for $\delta=0$, the estimator $T_{R P}$ turns out to be the product-type estimator

$T_{R 2}=\bar{y}\left(\frac{\bar{x} C_{x}+M_{d}}{\bar{X} C_{x}+M_{d}}\right)$

To the first degree of approximation the mean squared errors of $T_{R 2}, T_{p 2}$ are respectively given by

$\operatorname{MSE}\left(T_{R 2}\right)=\frac{(1-f)}{n} \bar{Y}^{2}\left[C_{y}^{2}+\theta C_{x}^{2}\{\theta-2 K\}\right]$
$\operatorname{MSE}\left(T_{P 2}\right)=\frac{(1-f)}{n} \bar{Y}^{2}\left[C_{y}^{2}+\theta C_{x}^{2}\{\theta+2 K\}\right]$

From (3.7), (5.5) and (5.6), we have

$\operatorname{MSE}\left(T_{R 2}\right)-\operatorname{MSE}\left(T_{R P}\right)=\frac{4(1-f)}{n} \theta \bar{Y}^{2} C_{x}^{2}[(1-\delta)(\delta \theta-K)]$

$\operatorname{MSE}\left(T_{P 2}\right)-\operatorname{MSE}\left(T_{R P}\right)=\frac{4(1-f)}{n} \theta \bar{Y}^{2} \delta C_{x}^{2}[\theta(1-\delta)+K]$

It follows from (5.7) and (5.8) that the ratio-product estimator $T_{R P}$ is more efficient than

(i) The ratio type estimator $T_{R 2}$ if

$\min \left(\frac{K}{\theta}, 1\right)<\delta<\max \left(\frac{K}{\theta}, 1\right)$

(ii) The product- type estimator $T_{P 2}$ if

$\min \left(\left(1+\frac{K}{\theta}\right), 0\right)<\delta<\max \left(\left(1+\frac{K}{\theta}\right), 0\right)$

Further, if we set $\left(C_{x}, M_{d}\right)=(1,0)$ in (5.3) and (5.4) the ratio-type estimator $T_{R 2}$ and product-type $T_{P 2}$ estimators respectively reduces to

$\mathrm{T}_{R 2} \rightarrow \bar{y} \frac{\bar{X}}{\bar{x}}$ (Usual ratio estimator)

$\mathrm{T}_{P 2} \rightarrow \bar{y} \frac{\bar{x}}{\bar{X}}$ (Usual product estimator)

Putting $\left(C_{x}, M_{d}\right)=(1,0)$ in (5.3) and (5.6) we get the mean squared errors of usual ratio and product estimators respectively as

$\operatorname{MSE}\left(T_{R 1}\right)=\frac{(1-f)}{n} \bar{Y}^{2}\left[C_{y}^{2}+C_{x}^{2}\{1-2 K\}\right]$

$\operatorname{MSE}\left(T_{P 1}\right)=\frac{(1-f)}{n} \bar{Y}^{2}\left[C_{y}^{2}+C_{x}^{2}\{1+2 K\}\right]$

From (3.7), (5.13) and (5.14) we have

$\operatorname{MSE}\left(T_{R 1}\right)-\operatorname{MSE}\left(T_{R P}\right)=\frac{(1-f)}{n} \bar{Y}^{2} C_{x}^{2}(1+\theta-2 \delta \theta)(1-\theta-2 K+2 \delta \theta)$

$\operatorname{MSE}\left(T_{P 1}\right)-\operatorname{MSE}\left(T_{R P}\right)=\frac{(1-f)}{n} \bar{Y}^{2} C_{x}^{2}(1-\theta+2 \delta \theta)(1+\theta+2 K-2 \delta \theta)$

From (5.15) and (5.16) we note that the ratio-product estimator $T_{R P}$ is better than

(i) The ratio type estimator $T_{R 1}$ if

$\min \left\{\left(\frac{1+\theta}{2 \theta}\right),\left(\frac{2 \mathrm{~K}+\theta-1}{2 \theta}\right)\right\}<\delta<\max \left\{\left(\frac{1+\theta}{2 \theta}\right),\left(\frac{2 \mathrm{~K}+\theta-1}{2 \theta}\right)\right\}$

(ii) The product- type estimator $T_{p 1}$ if

$\min \left\{\left(\frac{\theta-1}{2 \theta}\right),\left(\frac{2 \mathrm{~K}+\theta+1}{2 \theta}\right)\right\}<\delta<\max \left\{\left(\frac{\theta-1}{2 \theta}\right),\left(\frac{2 \mathrm{~K}+\theta+1}{2 \theta}\right)\right\}$ 
Bajopas Volume 10 Number 1 June, 2017

Estimator based on the Optimum value

The optimum value of $\delta$ in (3.8) is given by

$\delta_{0}=\frac{1}{2}\left(1+\frac{K}{\theta}\right)$

Where $\theta$ is a known quantity and

$K=\rho \frac{C_{y}}{C_{x}}=\rho \frac{S_{y} \bar{X}}{S_{x} \bar{Y}}=\frac{S_{x y}}{R S_{x}^{2}}=\frac{\beta}{R}$

Replacing $\beta$ and $R$ by their consistent estimators

$\hat{\beta}=\frac{s_{x y}}{s_{x}^{2}}$ and $\hat{R}=\frac{\bar{y}}{\bar{x}}$ respectively.

From (6.1) we get a consistent estimator of $\delta_{0}$ as

$\hat{\delta}_{0}=\frac{1}{2}\left(1+\frac{\hat{K}}{\theta}\right)$

Where $\hat{K}=\frac{\hat{\beta}}{\hat{R}}$

If the experimenter is unable to guess the value of $K$, then it is worth advisable to replace $K$ by $\widehat{K}$ in $(3.21)$

. Thus, the estimator based on the estimated 'optimum' value.

$\hat{T}_{R P O}=\frac{\bar{y}}{2}\left[\left(1+\frac{\hat{K}}{\theta}\right)\left(\frac{\bar{X} C_{x}+M_{d}}{\bar{x} C_{x}+M_{d}}\right)+\left(1+\frac{\hat{K}}{\theta}\right)\left(\frac{\bar{x} C_{x}+M_{d}}{\bar{X} C_{x}+M_{d}}\right)\right]$

To obtain the $M S E$ of $\widehat{T}_{R P O}$ we write

Let $e_{2}=\frac{(\hat{k}-K)}{K}$, then $\hat{k}=K\left(1+e_{2}\right)$

with $E\left(e_{2}\right)=K+o\left(n^{-1}\right)$, expanding (6.3) in terms of $e^{\prime} s$ we have

$\hat{T}_{R P O}=\frac{\bar{Y}}{2}\left(1+e_{0}\right)\left[\left\{1+\frac{K}{\theta}\left(1+e_{2}\right)\right\}\left(1+\theta e_{1}+\theta^{2} e_{1}^{2}+\theta^{3} e_{1}^{3}+\theta^{4} e_{1}^{4}+\ldots\right)+\left\{1-\frac{K}{\theta}\left(1+e_{2}\right)\right\}\left(1+\theta e_{1}\right)\right]$

From (6.4) we have

$$
\begin{aligned}
= & \frac{\bar{Y}}{2}\left(1+e_{0}\right)\left[1+\frac{K}{\theta}\left(1+e_{2}\right)-\theta e_{1}-K\left(e_{1}+e_{1} e_{2}\right)+K \theta\left(e_{1}^{2}+e_{2} e_{1}^{2}\right)+\ldots+1-\frac{K}{\theta}\left(1+e_{2}\right)+\theta e_{1}-K\left(e_{1}+e_{1} e_{2}\right)\right] \\
= & \frac{\bar{Y}}{2}\left(1+e_{0}\right)\left[2-2 K\left(e_{1}+e_{1} e_{2}\right)+K \theta\left(e_{1}^{2}+e_{2} e_{1}^{2}\right)+\ldots\right] \\
= & \bar{Y}\left(1+e_{0}\right)\left[1-K\left(e_{1}+e_{1} e_{2}\right)+\frac{K \theta}{2}\left(e_{1}^{2}+e_{2} e_{1}^{2}\right)+\ldots\right] \\
= & \bar{Y}\left(1+e_{0}\right)\left[1+e_{0}-K\left(e_{1}+e_{0} e_{1}+e_{1} e_{2}+e_{0} e_{1} e_{2}\right)+\frac{K \theta}{2}\left(e_{1}^{2}+e_{2} e_{1}^{2}+e_{0} e_{1}^{2}+e_{0} e_{2} e_{1}^{2}\right)+\ldots\right]
\end{aligned}
$$

Neglecting the terms of $e^{\prime} s$ having power greater than two we have

$$
\begin{aligned}
& \hat{T}_{R P O}=\bar{Y}\left[1+e_{0}-K e_{1}-K\left(e_{0} e_{1}+e_{1} e_{2}\right)+\frac{K \theta}{2} e_{1}^{2}\right] \text { or } \\
& \left(\hat{T}_{R P O}-\bar{Y}\right)=\bar{Y}\left[e_{0}-K e_{1}-K\left(e_{0} e_{1}+e_{1} e_{2}\right)+\frac{K \theta}{2} e_{1}^{2}\right]
\end{aligned}
$$

Now squaring both sides of (6.5) and neglecting the terms of $e^{\prime} s$ having power greater than the second we have

$$
\left(\hat{T}_{R P O}-\bar{Y}\right)^{2}=\bar{Y}^{2}\left[e_{0}^{2}+K^{2} e_{1}^{2}-2 K e_{0} e_{1}\right]
$$

Taking the expectation of both sides of (6.6) we get the mean square error (MSE) of $\widehat{T}_{R \mathrm{PO}}$ to the first order of approximation as 
Bajopas Volume 10 Number 1 June, 2017

$$
\begin{aligned}
& \operatorname{MSE}\left(\hat{T}_{R P O}\right)=E\left(\hat{T}_{R P O}-\bar{Y}\right)^{2}=\bar{Y}^{2} E\left[e_{0}^{2}+K^{2} e_{1}^{2}-2 K\left(e_{0} e_{1}\right)\right] \\
& =\frac{(1-f)}{n} \bar{Y}^{2}\left[C_{y}^{2}+K^{2} C_{x}^{2}-2 K \rho C_{y} C_{x}\right] \\
& =\frac{(1-f)}{n} \bar{Y}^{2}\left[C_{y}^{2}+\left(\rho \frac{C_{y}}{C_{x}}\right)^{2} C_{x}^{2}-2\left(\rho \frac{C_{y}}{C_{x}}\right) \rho C_{y} C_{x}\right] \\
& =\frac{(1-f)}{n} \bar{Y}^{2}\left(C_{y}^{2}-\rho^{2} C_{y}^{2}\right) \\
& =\frac{(1-f)}{n} \bar{Y}^{2} C_{y}^{2}\left(1-\rho^{2}\right) \\
& =\frac{(1-f)}{n} S_{y}^{2}\left(1-\rho^{2}\right)
\end{aligned}
$$

Which is equal to the minimum $M S E$ of $T_{R P}$ or the $M S E$ of $T_{R P O}$ given by (3.10). Thus, we established the result that the MSE of the estimator $\hat{\mathrm{T}}_{R P O}$ in (6.3) based on 'estimated optimum value' to the first degree of approximation is same as that of $\hat{\mathrm{T}}_{R P O}$ in (3.9) 134 teresting to note the estimator $\hat{\mathrm{T}}_{R P O}$ in (6.3) can be used as an alternative to the $\hat{\mathrm{T}}_{R P O}$ in (3.9) if the vuruc ur wie parameter is not known.

\section{Numerical Comparison}

\begin{tabular}{|c|c|c|}
\hline Parameters & Population 1: $Y=$ output $X=$ fixed capital & $\begin{array}{l}\text { Population 2: } Y=\text { output } X=\text { number } \\
\text { of workers }\end{array}$ \\
\hline$N$ & 80 & 80 \\
\hline$n$ & 20 & 20 \\
\hline $\bar{Y}$ & 51.8264 & 51.8264 \\
\hline $\bar{X}$ & 11.2646 & 2.8513 \\
\hline$\rho$ & 0.941 & 0.9150 \\
\hline$C_{y}$ & 0.3542 & 0.3542 \\
\hline$C_{x}$ & 0.7507 & 0.9484 \\
\hline$M_{d}$ & 7.575 & 1.4800 \\
\hline
\end{tabular}

To see the merit of the suggested estimator $T_{R P}$ over $\bar{y}, T_{R 1}, T_{P 1}, T_{R 2}$ and $T_{P 2}$.

Dataset: The populations considered in this study are real-life dataset comprises of population 1 and 2 . The population 1 is taken from Singh and Chaudary (1986) given in page 177 and population 2 is taken from Murthy (1964) given in page 228. Table 1 below summarized the dataset for the study.

\section{Table 1: Summary of the Dataset}

Therefore, we see the merit of the proposed estimators $T_{R P}$ over some existing Ratio and Product estimators $\bar{y}, T_{R 1}, T_{P 1}, T_{R 2}$ and $T_{P 2}$ in Table 2 and Table 3.

Table 2: Ranges of $\delta$ under which the proposed estimator $T_{R P}$ is better than $\bar{y}, T_{R 1}, T_{P 1}, T_{R 2}$ and $T_{P 2}$.

\begin{tabular}{cll}
\hline Populations $\rightarrow$ & 1 & 2 \\
\hline $\bar{y}$ & $0.5<\delta<1.340$ & $0.5<\delta<1.029$ \\
$T_{R 2}$ & $0.842<\delta<1.000$ & $0.529<\delta<1.000$ \\
$T_{P 2}$ & $0.00<\delta<1.842$ & $0.000<\delta<1.529$ \\
$T_{R 1}$ & $0.394<\delta<1.448$ & $0.255<\delta<1.274$ \\
$T_{P 1}$ & $-0.448<\delta<2.2896$ & $-0.274<\delta<1.802$ \\
$\delta$ & 0.921 & 0.764 \\
\hline
\end{tabular}


Table 3: The variance and MSE of $\bar{y}, T_{R 1}, T_{P 1}, T_{R 2}$ and $T_{P 2}$ and the proposed estimator $T_{R P}$

\begin{tabular}{cll}
\hline Populations $\rightarrow$ & 1 & 2 \\
\hline $\bar{y}$ & 12.637 & 12.637 \\
$T_{R 2}$ & 1.843 & 10.461 \\
$T_{P 2}$ & 55.018 & 90.498 \\
$T_{R 1}$ & 18.995 & 63.216 \\
$T_{P 1}$ & 119.804 & 143.252 \\
$T_{R P}$ & 1.447 & 2.057 \\
\hline
\end{tabular}

The Percentage relative efficiency (PRE) of different estimators $T$ respect to $\bar{y}$ is defined as, $\operatorname{PRE}(T ., \bar{y})=\frac{V(\bar{y})}{V(T .)} \mathrm{X} 100$

Table 4: Percentage relative efficiency of different estimators with respect to $\bar{y}$

\begin{tabular}{llllcll}
\hline $\begin{array}{c}\text { Populations } \\
\downarrow\end{array}$ & $\bar{y}$ & $T_{R 1}$ & $T_{P 1}$ & $T_{R 2}$ & $T_{P 2}$ & $T_{R P}$ \\
\hline 1 & 100 & $<100$ & $<100$ & 685.69 & $<100$ & 873.23 \\
2 & 100 & $<100$ & $<100$ & 120.80 & $<100$ & 614.35 \\
\hline
\end{tabular}

\section{DISCUSSION}

We have proposed an estimator of the ratio-product estimator and obtained the asymptotically optimum estimator (AOE) with its approximate MSE formula for the proposed estimator using the coefficient of variation and median of the auxiliary variable $X$ in simple random sampling.

Theoretically, we have demonstrated that the proposed estimator is always more efficient than other estimators $\bar{y}, T_{R 1}, T_{P 1}, T_{R 2}$ and $T_{P 2}$ under the effective ranges of $\delta$ and its optimum values.

In addition, we support these theoretical results numerically using the data sets as shown in Table 1.

Table 2 provides the wide ranges of $\delta$ along with its optimum values for which the proposed estimator $T_{R P}$ is more efficient than other estimators $\bar{y}, T_{R 1}$ ，
$T_{P 1}, T_{R 2}$ and $T_{P 2}$ as far as mean squared error criterion is considered. It is also observed from Table $\mathbf{2}$ that there is a scope for chosen $\delta$ to obtain better estimators than $\bar{y}, T_{R 1}, T_{P 1}, T_{R 2}$ and $T_{P 2}$.

Table 4 provides that there is a considerable gain in efficiency by using proposed estimator $T_{R \mathrm{P}}$ over the estimators $\bar{y}, T_{R 1}, T_{P 1}, T_{R 2}$ and $T_{P 2}$. This shows that even if the scalar $\delta$ deviates from its optimum values $\left(\delta_{\text {opt }}\right)$. The suggested estimator $T_{R P}$ will yield better estimate than $\bar{y}, T_{R 1}, T_{P 1}, T_{R 2}$ and $T_{P 2}$.

$$
\begin{aligned}
& \operatorname{MSE}(\bar{y})-\operatorname{MSE}\left(T_{R P}\right)=\frac{(1-f)}{n} \bar{Y}^{2} C_{y}^{2}>0, \\
& \operatorname{MSE}\left(T_{R 1}\right)-\operatorname{MSE}\left(T_{R P}\right)=\frac{(1-f)}{n} \bar{Y}^{2} C_{x}^{2}(1+K)^{2}>0 \\
& \operatorname{MSE}\left(T_{P 1}\right)-\operatorname{MSE}\left(T_{R P}\right)=\frac{(1-f)}{n} \bar{Y}^{2} C_{x}^{2}(1-K)^{2}>0 \\
& \operatorname{MSE}\left(T_{R 2}\right)-\operatorname{MSE}\left(T_{R P}\right)=\frac{(1-f)}{n} \bar{Y}^{2} C_{x}^{2}(\theta+K)^{2}>0 \\
& \operatorname{MSE}\left(T_{P 2}\right)-\operatorname{MSE}\left(T_{R P}\right)=\frac{(1-f)}{n} \bar{Y}^{2} C_{x}^{2}(\theta-K)^{2}>0
\end{aligned}
$$




\section{CONCLUSION}

Evidence from the study revealed that the proposed estimator is more efficient than the already existing ratio, product and ratio- product type estimators based on some certain conditions and efficiency conditions. Therefore, there is always need to ensure that the auxiliary variable is highly correlated with the study variable and the population under consideration is homogeneously distributed, and when there is no correlation between the auxiliary variable and the

\section{REFERENCES}

Cochran, W.G. (1940). The estimation of the yield of the cereal experiments by sampling for ratio of grain to total produce. The journal of Agricultural Science,30: 262-275.

Das, A.K. and Tripathi, T.P. (1981). A class of sampling strategies for population mean using information on mean and variance of an auxiliary character. Proceedings of Italian Statistical Institute, Golden Jubilee International Conference of Statistics. Applications and new directions,174-181.

Housila, P.S. and Neha-Agnihotri (2008). A general procedure of estimating population mean using auxiliary information in sample surveys. Statistics in Transition-new series, 9(1):71-87.

Murthy, M.N. (1964). Product method of estimation, Sankhya A, 26:69-74.

Murthy, M. N. (1967). Sampling Theory and Methods. Calcutta, India: Statistical Publishing Society.

Okafor, F. C. (2002). Sample Survey Theory and Applications ( $1^{\text {st }}$ edition). Nsukka, Nigeria.

Perri, P.F. (2005). Combination of auxiliary variables in ratio-cum-product type estimators. In: Proceedings of Italian Statistical Society, Intermediate meeting on Statistics and Environment, Messina, Italy, September 2005,pp. 193-196.

Reddy, V.N. and Singh, H.P. (1978). A study on the use of prior knowledge on certain population study variable, the application of single- phase sample will not yield more efficient or the mean per unit will be more efficient.

Hence we conclude that the proposed class of estimator $T_{\mathrm{RP}}$ is more efficient than the other estimators in case of its optimality in simple random sampling. Thus, it is preferred to use the proposed estimator $T_{R \mathrm{P}}$ in practice over $\bar{y}, T_{R 1}, T_{\mathrm{P} 1}, T_{R 2}$ and $T_{\mathrm{P} 2}$.

parameters in estimation, Sankhya C, 40: 2937.

Singh, H.P. and Tailor, R. (2003). Estimation of finite population mean using known correlation coefficient of auxiliary characters. Statistica, Anno LXV, 4:407-418.

Singh, H.P. and Tailor, R. (2005). Estimation of finite population mean with known coefficient of variation of auxiliary characteristic. Statistica, anno LXV, (3):301-313.

Singh, D. and Chaudary, F.S. (1986). Theory and analysis of sample survey Designs. New Age International Publisher.pp. 177-178.

Singh, H.P. and Ruiz Espejo, M. (2003). On linear regression and ratio-product estimation of a finite population mean. The Statistician, 52(1): 59-67.

Sisodia B.V.S. and Dwivedi, V.K. (1981). A modified ratio estimator using coefficient of variation of auxiliary variable. Journal of Indian Society of Agricultural Statistics. 33(1) :1318.

Srivenkataramana, T. and Tracy, D. S. (1980). On ratio and product methods of estimation in sampling. Statistica Neerlandica, 33: 37-49.

Subramani, J. and Kumarapandiyan, G. (2012). Estimation of population mean using coefficient of variation and median of the auxiliary variable. International Journal of Probability Statistics, 1(4): 111-118, DOI $\underline{10.5923 / j . ~ i j p s .20120104 .04 . ~}$ 